\title{
HIDRADENOMA PAPILÍfERO PERIANAL
}

\author{
Oscar Mora Hernández MD*, Luisa Castaño Ortiz MD**
}

\section{Resumen}

El hidradenoma papilífero es una neoplasia benigna, quística y papilar, poco frecuente que por lo general aparece en la vulva, aunque también se ha descrito en otras zonas. Presentamos el caso de una mujer de 74 años con uno localizado en región perianal, cuyo diagnóstico clínico inicial fue sarcoma de Kaposi. El interés de este informe radica en recalcar que esta afección puede aparecer en una zona diferente a la vulva, para evitar diagnósticos erróneos.

Palabras clave: hidradenoma papilífero, vulva, región perianal.

\section{PERIANAL PAPILLARY HIDRADENOMA}

\section{Abstract}

Papillary hidradenoma is a rare benign neoplasm characterized by the presence of cystic and papillary structures that usually occurs on the vulva but has also been described in other locations. We report the case of a 74 year-old woman with a perianal papillary hidradenoma. An initial diagnosis of Kaposi's sarcoma was made. In order to prevent mistaken diagnoses, this report aims to emphasize this lesion is not limited to the vulva and may appear in other locations.

Key words: papillary hidradenoma, vulva, perianal region

Fecha recibido: mayo 8 de 2012. Fecha aceptado: mayo 31 de 2012

* Dermatólogo, Hospital de San José. Instructor Asistente, Fundación Universitaria de Ciencias de la Salud. Bogotá DC, Colombia.
** Residente II de Dermatología, Fundación Universitaria de Ciencias de la Salud. Bogotá DC. Colombia. 


\section{Intreducción}

El hidradenoma papilífero es una neoplasia benigna que se origina en las glándulas sudoríparas apocrinas vulvares y anogenitales, ocurre en mujeres entre 20 y 80 años de edad ${ }^{1-4}$, a menudo es un nódulo solitario de cerca de $1 \mathrm{~cm}$ de diámetro, bien delimitado y del color de la piel normal, aunque puede ser translúci$\mathrm{do}^{3}$. La localización mas frecuente es la vulva, pero también se ha descrito en labios mayores y menores, surco interlabial, clítoris, horquilla posterior, monte de venus, periné y ano. ${ }^{4-8}$ Hay casos reportados en parpados y pezón. ${ }^{5}$

\section{Caso clínico}

Mujer de 74 años con historia de hipertensión arterial controlada sin otro antecedente de importancia. Acude a la consulta de dermatología por presentar lesión en región perianal de seis meses de evolución, que ha ido aumentando de tamaño y genera dolor con la deposición y la fricción. A la exploración física se observa lesión nodular violácea con telangiectasias en su superficie de $1 \mathrm{~cm}$ de diámetro, en región perianal (Figura 1). Se decide resección quirúrgica con impresión diagnóstica de sarcoma de Kaposi

En el servicio de patología se recibió una losange de piel que mide $2 \times 1.5 \times 0.6 \mathrm{~cm}$ donde se identifica una lesión elevada, blanquecina, de bordes regulares y superficie lisa, de $1 \times 1 \mathrm{~cm}$, reparada y orientada. En la histología se observó una formación quística apocrina benigna constituida por estructuras papilares y grandes túbulos revestidos por una capa superficial de epitelio cilíndrico y otra mioepitelial basal asociada con estroma fibroso (Figura 2). Todos los bordes se encontraron libres de neoplasia, sin necrosis, atipia ni mitosis. No fue necesario el estudio de inmunohistoquímica.

\section{Discusión}

Se trata de una neoplasia benigna quística y papilar poco frecuente que aparece en las zonas vulvar y ano- genital de mujeres entre 20 y 80 años ${ }^{2}$. Es infrecuente en la raza negra. ${ }^{3}$ Corresponde a un nódulo o pólipo de crecimiento exofítico único, de aparición unilateral que puede sangrar y ulcerarse llegando a simular un carcinoma. Al microscopio está formado por túbulos elongados y papilas grandes que le confieren un aspecto arboriforme. Las papilas tienen un eje central y dos capas de células: una mioepitelial y otra de luminales columnares con secreción por decapitación. ${ }^{4}$

La secreción apocrina luminal de la cavidad se aprecia como un material homogéneo eosinófilo. El estroma está constituido por tejido fibroso comprimido que se halla separado de la dermis por hendiduras. Salvo casos puntuales no existe conexión con el epitelio superficial, se puede observar algún neutrófilo en el tejido conectivo ${ }^{4}$ y no se ven células plasmáticas en el eje papilar. Las células epiteliales que tapizan las

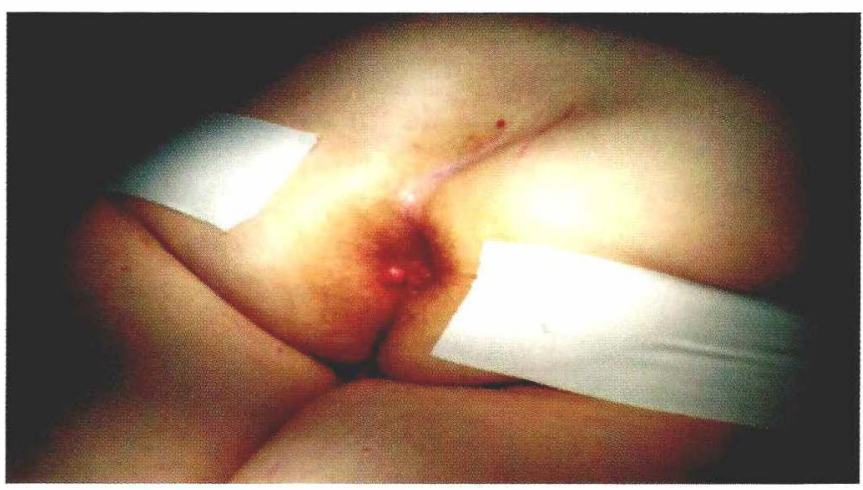

Figura I. Aspecto macroscópico del hidradenoma papilifero perianal antes de la cirugía.



Figura 2. Aspecto microscópico del hidradenoma papilífero. 
papilas se tiñen con citoqueratinas de bajo peso molecular. El borde luminal es positivo para EMA, CEA y GCDFP-15 y negativo para la proteína S-100 y citoqueratinas de alto peso.

La histogénesis de este tumor no es clara, aunque parece proceder de glándulas sudoríparas anogenitales o de tipo mamario. ${ }^{5}$ La descripción del primer caso se remonta a 1878 por Werth, que lo define como un tumor quístico con un epitelio cilíndrico que comparte similitudes anatomopatológicas con el adenoma papilar de mama. ${ }^{1}$ En 1941, McDonald sugiere por primera vez la posibilidad de que tenga una secreción apocrina ${ }^{2}$. En el año 1991 Vander Putte describió estas glándulas anogenitales, que se localizan en las áreas interlabial, posterior paramediana al periné y perianal, con características apocrinas. ${ }^{6}$

La similitud entre algunos tumores apocrinos y el hidradenoma papilífero sugiere que pueden tener la misma histogénesis a ser el mismo tumor con escasas expresiones diferentes cuando se encuentra en genitales femeninos o masculinos. ${ }^{7}$ Puede confundirse con una metástasis de un carcinoma papilar o un siringocistoadenocarcinoma papilífero, que puede adoptar diversas variantes morfológicas, algunas muy parecidas al tumor en mención. ${ }^{8-9}$ En conclusión el conocimiento de esta lesión evitará el diagnóstico erróneo.

\section{Referencias}

1. Werth R. ZurAnatomie der Cysten der vulva. Zentralb fGynakol. 1878; 22: 513-6.

2. McDonald J.R. Apocrine sweat gland carcinoma of the vulva. Am J ObstetricGynecology. 1941; 42:304-9.

3. Ollague J, Torres M. Neoplasias de glándulas sudoríparas. Dermatol Perú. 2005; $15(2): 211-21$

4. QueipoGutierrez FJ, Pardo J. Hidradenomapapiliferum of theanus. RevEsp Patol.2010; 43(4):233-35.

5. Guiote-Domínguez MV, Serrano-Falcón MM, Linares-Solano J, Burkhardt-Pérez $\mathrm{P}$, Serrano-Ortega S. Naranjo-Sintes R. Lesión nodular en la región vulvar. Actas Dermosifiliogr. 2007; 98(6):435-64.

6. Handa Y, Yamanaka N, Inagaki H, Tomita Y. Large ulcerated perianal hidradenomapapilliferum in a young female. DermatolSurg. 2003; 29:790-2.

7. Petter CY, Humphreys T, Goldberg L. Hidrocistomaapocrino: diagnóstico diferencial con otras lesiones pigmentadas de la piel. DermVenez. 1995; 33:131-34.

8. Kazakov DV, Requena L, Kutzner H. Fernández-Figueras MT, Kacerovska D, Mentzel T, et al. Morphologic diversity of Syringocystadenocarcinomapapilliferum based on a clinicopathologic study of 6 cases and review of the literature. Am J Dermatopathol. 2010; 32(4):340-7.

9. Daniel F, Mahmoudi A, de Parades V, Flejou JF, Atienza P. An uncommon Perianal nodule: hidradenomapapilliferum. GastroenterolClin Biol. 2007 Feb; 31(2): 166-8. 